\title{
ENSEÑANZA VIRTUAL DE LA BIOÉTICA. DESAFÍOS
}

\author{
Eduardo Rodríguez Yunta, Carolina Valdebenito Herrera y Fernando Lolas Stepke*
}

Resumen: El presente artículo reflexiona sobre la nueva modalidad de enseñanza en bioética utilizando una plataforma de aprendizaje virtual. Se señalan las ventajas y dificultades de este nuevo modelo y los desafíos para que sea más efectivo en sus objetivos pedagógicos. Se recomienda el uso de modelos de aprendizaje semipresenciales o de combinación mixta presencial y no presencial en la utilización de la enseñanza virtual en bioética.

Palabras clave: bioética, enseñanza virtual

\section{BIOETHICS' VIRTUAL TEACHING. CHALLENGES}

\begin{abstract}
The present paper reflects about the new bioethics teaching modality that employs a platform of virtual teaching. It points out this model's advantages and difficulties and the challenges it faces in order to reach an effective result of its pedagogical aims. It recommends to employ semipresential learning models or the combination of a mixed bioethics' virtual teaching: presential and non presential.
\end{abstract}

Key words: bioethics, virtual teaching

\section{ENSINO VIRTUAL DA BIOÉTICA. DESAFIOS}

Resumo: O presente artigo reflete sobre a nova modalidade de ensino em bioética, utilizando uma plataforma de aprendizagem virtual. Assinalam-se as vantagens e dificuldades deste novo modelo e os desafios para que seja mais efetivo em seus objetivos pedagógicos. Recomenda-se o uso de modelos de aprendizagem semipresenciais ou de combinação mista presencial e não presencial, na utilização do ensino virtual em bioética.

Palavras chave: bioética, ensino virtual 


\section{Introducción}

Debido al avance en las tecnologías de la comunicación, en los últimos años se han generado numerosas iniciativas para explorar las ventajas de ofrecer cursos virtuales de formación en bioética. La enseñanza virtual es un proceso de enseńanza a distancia (no presencial) basado en una plataforma de aprendizaje cooperativa, con nuevas oportunidades en recursos didácticos y en un nuevo modelo educativo centrado en el alumno. Para la formación en bioética es esencial desarrollar habilidades de debate/diálogo y reflexión/deliberación, base de la resolución de conflictos morales, y los espacios virtuales proporcionan formas particulares de realizar esta interacción. Teniendo en cuenta que la bioética es cada vez más necesaria en la práctica profesional, sobre todo en el campo de la salud y de la investigación científica, y que la modalidad plenamente presencial se vuelve difícil de conciliar con horarios y responsabilidades de los profesionales, la modalidad virtual aparece como una alternativa en la presente situación.

En el nuevo entorno social, una de las actividades más afectadas por las nuevas tecnologías de la información y las comunicaciones es la educación. La innovación tecnológica-característica de la sociedad actual- desarrolla un papel fundamental en todos los niveles educativos -y en particular en la universidad- con la incorporación de sistemas de información, herramientas de comunicación y elementos multimedia. Este enfoque educativo intenta que los alumnos, más que adquirir grandes cantidades de información, sean capaces de buscarla, seleccionarla, interpretarla, relacionarla semánticamente con sus conocimientos previos y comunicarla.

Los resultados de estudios realizados en 2004 por la UNESCO en América Latina indican que la tendencia que se manifiesta con mayor fuerza es hacia una articulación de lo presencial con lo distante y lo virtual con lo no-virtual(1). En la actualidad, la mayoría de los centros de educación superior están inmersos en el desarrollo del componente no presencial de una buena parte de sus materias, mediante el establecimiento de campus virtuales para los cuales se encuentran disponibles distintas plataformas. Los entornos virtuales de enseñanza-aprendizaje proporcionan un soporte fundamental para adecuar la metodología docente a las necesidades de los alumnos, ofreciendo ciertas ventajas, pero esto requiere una serie de cambios en estudiantes, profesores e instituciones.
En la Web están surgiendo una serie de programas virtuales de educación en bioética, que pueden tener mayor o menor utilidad para el usuario y con una amplia variedad en cuanto a costos y grados de interacción entre docente y alumno, y entre los mismos estudiantes; sin embargo, es difícil saber de antemano el nivel de calidad de estos programas y con cuánta aceptación social cuenta su currículo.

\section{Nuevo modelo educativo}

La enseńanza virtual se basa en un nuevo modelo educativo, en el cual el aprendizaje no es una copia o reproducción pasiva de la información recibida, sino que requiere un proceso activo de interacción y relación de los nuevos contenidos con los que ya se poseían. Según el modelo constructivista, el aprendizaje consiste en un proceso personal de construcción de nuevos conocimientos a partir de experiencias pasadas y presentes y no en la simple acumulación de conocimiento: "el aprendizaje se forma construyendo nuestros propios conocimientos desde nuestras propias experiencias"(2). Este modelo encaja bien con el de la formación en bioética, que debe centrar su interés en la adquisición de valores, toma de conciencia, sensibilización e interacción o diálogo entre los participantes.

Teniendo en cuenta que el discurso bioético es integrador y dialogante, la educación en bioética debe adaptarse a esta forma. La bioética alberga una pluralidad de voces, una diversidad de fines y una extensa utilización del diálogo como herramienta social, mediando entre racionalidades, personas, instituciones y disciplinas para formular problemas, articular disensos y llegar a consensos(3). La bioética tiende un puente que hace accesible el mundo del experto al lego. En la educación, por tanto, el paso de la lección expositiva a la discusión, de la explicación al diálogo guiado, introduce un enfoque especialmente deseable en la formación bioética accesible a la modalidad virtual. Una de las principales aplicaciones a la enseñanza virtual es el aprendizaje colaborativo, cuya premisa básica es la construcción del consenso a través de la cooperación.

A este respecto, el espacio virtual posibilita el diálogo entre el docente y el alumno y se puede extender a la interacción de alumno con alumno para que se produzca aprendizaje de una forma independiente y cooperativa. 
El modelo se sustenta en dos pilares básicos:

- Dimensión tecnológica: el material didáctico digital accesible desde la Web, que integra los elementos básicos del proceso de autoaprendizaje, con introducciones, objetivos, contenidos, actividades, ejercicios de autoevaluación, glosarios, etc. Es importante aprovechar la potencialidad de Internet como canal de comunicación en el diseño de los contenidos, de forma que incluya interactividad, uso de multimedia y acceso a información actualizada en tiempo real.

- Dimensión didáctica: los materiales de aprendizaje estructurados desde un punto de vista pedagógico y de un modo adecuado al proceso de autoaprendizaje realizado por el alumno, poniendo énfasis en el apoyo personalizado.

La figura del docente también sufre un cambio en este modelo, ya que su función pasa a ser más la de un tutor y/o asesor con las siguientes responsabilidades:

- Organizar el curso.

- Aportar información como especialista.

- Motivar al alumno con las herramientas de Internet disponibles, resolviendo dudas, asesorando y fomentando trabajos grupales.

\section{Características de la enseńanza virtual}

En la adecuación al modelo virtual, la forma de aprendizaje posee características propias:

- Aprendizaje sin coincidencia profesor-alumno en el espacio y el tiempo.

- El alumno pasa de ser un receptor de conocimiento a interpretar un papel activo en el proceso de aprendizaje (proceso de autoformación).

- El docente pasa a ser un facilitador de contenidos con capacidad para tutelar y/o asesorar.

- La enseńanza virtual requiere de un cambio en el modelo educativo clásico para adaptarse al nuevo entorno de aprendizaje no presencial y basado en las tecnologías de la información.

- Los aspectos más directamente afectados por el uso de los medios digitales son: la presentación de la información del docente a los alumnos, el material de estudio y consulta, la interacción docente-alumno y el propio proceso de aprendizaje y autoevaluación del alumno.

Los programas online en español para la enseñanza de la bioética y temas afines presentan mucha variedad en metodología y número de horas exigidos. Algunos de estos programas son los siguientes:

Programas online de bioética

\begin{tabular}{|c|c|c|c|c|}
\hline Temática bioética & Gestor & Título & Sitio web & Metodología \\
\hline $\begin{array}{l}\text { Fundamentación, } \\
\text { inicio y final de la vida, } \\
\text { salud, biotecnología, } \\
\text { genética }\end{array}$ & $\begin{array}{l}\text { Institut Borja de Bioética } \\
\text { (IBB), Universitat Ramon } \\
\text { Llull, España }\end{array}$ & $\begin{array}{l}\text { Máster, } 60 \\
\text { créditos, } 700 \text { hrs. }\end{array}$ & $\begin{array}{l}\text { www.ibbioetica.org / www.bioetica- } \\
\text { debat.org }\end{array}$ & $\begin{array}{l}\text { Presencial y online. } \\
\text { Ensayos, } \\
\text { evaluación, foro }\end{array}$ \\
\hline $\begin{array}{l}\text { Fundamentación, } \\
\text { enfermería, inicio y } \\
\text { final de la vida, comités } \\
\text { asistenciales, salud } \\
\text { pública, biotecnología }\end{array}$ & $\begin{array}{l}\text { UNIZAR, Universidades de } \\
\text { País Vasco / UPV-EHU, } \\
\text { La Laguna, Las Palmas de } \\
\text { Gran Canaria, Zaragoza, } \\
\text { Rovira i Virgili, Espańa }\end{array}$ & $\begin{array}{l}\text { Máster, } 50 \\
\text { créditos } 600 \text { hrs. }\end{array}$ & $\begin{array}{l}\text { www.emagister.com/Máster- } \\
\text { interuniversitario-bioetica-cursos- } \\
\text { 2464357.htm }\end{array}$ & $\begin{array}{l}\text { Semipresencial, } \\
\text { tutoría }\end{array}$ \\
\hline $\begin{array}{l}\text { Fundamentación, } \\
\text { salud mental, clínica }\end{array}$ & $\begin{array}{l}\text { Universidad de León e IAEU, } \\
\text { Barcelona, España }\end{array}$ & $\begin{array}{l}\text { Máster, } 50 \\
\text { créditos, } 500 \text { hrs. }\end{array}$ & $\begin{array}{l}\text { www.salud-mental.net/Máster- } \\
\text { salud-mental-b } \\
\text { ioetica.html }\end{array}$ & $\begin{array}{l}\text { Seminarios de casos } \\
\text { prácticos, tutorías, } \\
\text { supervisiones } \\
\text { clínicas y debates } \\
\text { del Campus } \\
\text { Virtual }\end{array}$ \\
\hline $\begin{array}{l}\text { Fundamentación, inicio } \\
\text { y final de la vida, clínica, } \\
\text { derecho sanitario }\end{array}$ & $\begin{array}{l}\text { Universidad de Murcia } \\
\text { (SUMA), Espańa }\end{array}$ & $\begin{array}{l}\text { Máster, } 50 \\
\text { créditos, } 500 \text { hrs. }\end{array}$ & $\begin{array}{l}\text { http://www.um.es/estudios/cursos/ } \\
\text { bioetica/ }\end{array}$ & $\begin{array}{l}\text { Tutoría, } \\
\text { cuestionarios, } \\
\text { ensayos }\end{array}$ \\
\hline $\begin{array}{l}\text { Fundamentación, } \\
\text { Derecho, inicio y final } \\
\text { de la vida }\end{array}$ & $\begin{array}{l}\text { Universidad Nacional de } \\
\text { Educación a Distancia UNED, } \\
\text { España }\end{array}$ & $\begin{array}{l}\text { Experto } \\
\text { Universitario } \\
\text { en Bioética, } 50 \\
\text { créditos, } 500 \text { hrs. }\end{array}$ & $\begin{array}{l}\text { www.fundacion.uned.es/cursos/ } \\
\text { humanidades/experto-universitario/ } \\
\text { bioetica }\end{array}$ & \begin{tabular}{|l|} 
Lecturas, tutoría, \\
trabajo dirigido, \\
resolución de casos, \\
tests
\end{tabular} \\
\hline
\end{tabular}




\begin{tabular}{|c|c|c|c|c|}
\hline $\begin{array}{l}\text { Fundamentación, } \\
\text { bioderecho, biomedicina } \\
\text { y derecho, investigación, } \\
\text { comités }\end{array}$ & $\begin{array}{l}\text { Universidad Nacional de } \\
\text { Educación a Distancia UNED, } \\
\text { España }\end{array}$ & \begin{tabular}{|l} 
Experto \\
Universitario \\
en Bioética y \\
Derecho, 50 \\
créditos, 500 hrs.
\end{tabular} & $\begin{array}{l}\text { www.uned.es/dpto_con/experto/ } \\
\text { bioetica_bioderecho.htm }\end{array}$ & $\begin{array}{l}\text { Lecturas, tutoría, } \\
\text { trabajo dirigido, } \\
\text { resolución de casos, } \\
\text { tests }\end{array}$ \\
\hline $\begin{array}{l}\text { Fundamentación, } \\
\text { clínica, } \\
\text { comités asistenciales y de } \\
\text { investigación }\end{array}$ & $\begin{array}{l}\text { Universidad de La Laguna y } \\
\text { Universidad de Las Palmas de } \\
\text { Gran Canaria, Espańa }\end{array}$ & $\begin{array}{l}\text { Máster, } 60 \\
\text { créditos, } 600 \text { hrs. }\end{array}$ & www.ebioetica.net & $\begin{array}{l}\text { Semipresencial. } \\
\text { Lecturas, tutoría, } \\
\text { videoconferencias, } \\
\text { ensayos }\end{array}$ \\
\hline $\begin{array}{l}\text { Fundamentación, } \\
\text { dignidad de la vida } \\
\text { humana, inicio y fin de } \\
\text { la vida, investigación, } \\
\text { genética, sexualidad }\end{array}$ & $\begin{array}{l}\text { Universidad Católica San } \\
\text { Antonio (UCAM), Murcia, } \\
\text { Espańa }\end{array}$ & $\begin{array}{l}\text { Máster, } 60 \\
\text { créditos, } 600 \text { hrs. }\end{array}$ & $\begin{array}{l}\text { http://www.ucam.edu/Máster_ } \\
\text { oficial/bioetica/index.htm }\end{array}$ & $\begin{array}{l}\text { Presencial, } \\
\text { semipresencial u } \\
\text { online, tutoría, } \\
\text { ensayos }\end{array}$ \\
\hline $\begin{array}{l}\text { Fundamentación, } \\
\text { clínica, comités } \\
\text { asistenciales }\end{array}$ & $\begin{array}{l}\text { Universidad Católica de } \\
\text { Valencia "San Vicente Mártir", } \\
\text { España }\end{array}$ & $\begin{array}{l}\text { Máster, } 75 \\
\text { créditos, } \\
700 \text { hrs. }\end{array}$ & www.ucv.es/bioetica & $\begin{array}{l}\text { Semipresencial y } \\
\text { online } \\
\text { Tutoría, plataforma } \\
\text { digital Ucvnet, } \\
\text { ensayos }\end{array}$ \\
\hline $\begin{array}{l}\text { Fundamentación, } \\
\text { clínica, } \\
\text { investigación }\end{array}$ & $\begin{array}{l}\text { Facultad de Medicina } \\
\text { Universidad de Buenos Aires, } \\
\text { Argentina }\end{array}$ & $\begin{array}{l}\text { Certificado }(200 \\
\text { hrs.) }\end{array}$ & http://www.fmv-uba.org.ar/cp & $\begin{array}{l}\text { Lecturas, } \\
\text { análisis de casos, } \\
\text { cuestionarios, } \\
\text { foros de discusión }\end{array}$ \\
\hline $\begin{array}{l}\text { Fundamentación, inicio } \\
\text { y final de la vida, clínica, } \\
\text { investigación }\end{array}$ & $\begin{array}{l}\text { Facultad de Medicina, } \\
\text { Pontificia Universidad Católica } \\
\text { de Chile }\end{array}$ & $\begin{array}{l}\text { Diploma (170 } \\
\text { hrs.) }\end{array}$ & $\begin{array}{l}\text { http://escuela.med.puc.cl/deptos/ } \\
\text { Bioética/DocenciaBioetica.html }\end{array}$ & $\begin{array}{l}\text { Semipresencial, } \\
\text { lecturas, análisis de } \\
\text { casos, ensayos }\end{array}$ \\
\hline $\begin{array}{l}\text { Bioética y derecho, } \\
\text { salud, } \\
\text { biotecnología }\end{array}$ & $\begin{array}{l}\text { Observatori de Bioética i Dret } \\
\text { Universidad de Barcelona, } \\
\text { España }\end{array}$ & $\begin{array}{l}\text { Máster } \\
50 \text { créditos, } \\
500 \text { hrs. } \\
\end{array}$ & http://www.bioeticayderecho.ub.es & $\begin{array}{l}\text { Semipresencial. } \\
\text { Campus virtual }\end{array}$ \\
\hline $\begin{array}{l}\text { Salud, } \\
\text { ética profesional, } \\
\text { comités, } \\
\text { investigación }\end{array}$ & $\begin{array}{l}\text { Facultad de Medicina, } \\
\text { Universidad de Chile }\end{array}$ & $\begin{array}{l}\text { Certificado } \\
\text { Módulos }\end{array}$ & info@medichi.cl & $\begin{array}{l}\text { Semipresencial. } \\
\text { Campus virtual, } \\
\text { talleres presenciales } \\
\text { locales, ensayos, } \\
\text { presentaciones } \\
\text { grupales }\end{array}$ \\
\hline $\begin{array}{l}\text { Ética de la investigación, } \\
\text { clínica y social }\end{array}$ & UNESCO y REDBIOETICA & Certificado & www.redbioetica-edu.com.ar & Tutoría \\
\hline $\begin{array}{l}\text { Fundamentación, } \\
\text { atención de salud, inicio } \\
\text { y final de la vida, } \\
\text { comités asistenciales, } \\
\text { derecho sanitario } \\
\end{array}$ & $\begin{array}{l}\text { SESCAM y Escuela Nacional } \\
\text { de Sanidad, Instituto de Salud } \\
\text { Carlos III, España }\end{array}$ & $\begin{array}{l}\text { Diploma, } 500 \\
\text { hrs. }\end{array}$ & $\begin{array}{l}\text { http://sescam.jccm.es/web/ } \\
\text { gestion/eventos/II_DIPLOMA_ } \\
\text { SUPERIOR_BIOETICA } \\
\text { secretaria.cursos@isciii.es }\end{array}$ & $\begin{array}{l}\text { Semipresencial. } \\
\text { Lecturas, talleres, } \\
\text { ensayos }\end{array}$ \\
\hline Ética de la investigación & $\begin{array}{l}\text { Family Health International } \\
\text { (FHI), OPS/OMS }\end{array}$ & Certificado & $\begin{array}{l}\text { http://www.fhi.org/sp/RH/ } \\
\text { Training/trainmat/ethicscurr/index. } \\
\text { htm }\end{array}$ & $\begin{array}{l}\text { Presencial o virtual. } \\
\text { Lecturas, } \\
\text { estudios de casos, } \\
\text { evaluaciones }\end{array}$ \\
\hline Comités asistenciales & $\begin{array}{l}\text { Instituto de Consulta y } \\
\text { Especialización en Bioética } \\
\text { (ICEB) }\end{array}$ & Diploma, 60 hrs. & www.bioeticacs.org & $\begin{array}{l}\text { Tutoría, análisis de } \\
\text { casos, tests }\end{array}$ \\
\hline $\begin{array}{l}\text { Fundamentación, } \\
\text { clínica, inicio y final de } \\
\text { la vida, dignidad de la } \\
\text { vida humana }\end{array}$ & $\begin{array}{l}\text { DBIO, Universidad Libre } \\
\text { Internacional de las Américas }\end{array}$ & $\begin{array}{l}\text { Diploma } 20 \\
\text { créditos, } 220 \text { hrs. }\end{array}$ & dbio@ulia.org & $\begin{array}{l}\text { Tutoría, lecturas, } \\
\text { análisis de casos }\end{array}$ \\
\hline
\end{tabular}




\begin{tabular}{|l|l|l|l|l|}
\hline Ética de la investigación & $\begin{array}{l}\text { Collaborative International } \\
\text { Training Initiative, University } \\
\text { of Miami, Estados Unidos, } \\
\text { Plataforma Latinoamericana } \\
\text { CIEB Universidad de Chile }\end{array}$ & Certificado & www.citiprogram.org/ & Lecturas, tests \\
\hline
\end{tabular}

\section{Ventajas y beneficios de la enseńanza virtual}

La generación de un espacio virtual posibilita una serie de ventajas y beneficios desde el punto de vista del aprendizaje:

- Permite enviar a cada participante, sin costo adicional, la información necesaria básica, a partir de la cual se inician las reflexiones y la construcción colaborativa del conocimiento.

- Proporciona un medio eficaz para la introducción de pedagogías y estrategias novedosas de enseñanzaaprendizaje por el uso de recursos multimedia.

- Promueve un modelo de aprendizaje activo con la construcción colaborativa de conocimiento.

- Amplía posibilidades para el contacto sincrónico y asincrónico entre el docente y el alumno y entre los alumnos, gracias a las herramientas de comunicación: correo electrónico, chat, videoconferencia, pizarra electrónica, etc.

- En la modalidad asincrónica, permite disponer los materiales de aprendizaje en el momento en que el alumno los necesita, respetándose su ritmo de aprendizaje. Igualmente, la resolución de dudas se puede realizar de forma personalizada y extendiéndose en el tiempo.

- Permite aprovechar mejor el tiempo del alumno y el que invierte el profesor en tareas expositivas, pudiéndose dedicar así más tiempo a la práctica.

- Posibilita el seguimiento del curso a distancia por parte de alumnos alejados geográficamente o con dificultad para el desplazamiento. Los materiales son accesibles vía Internet en cualquier momento y desde cualquier lugar.

- Permite la modalidad semipresencial con una proporción presencialidad/virtualidad variable según las necesidades.

- Favorece el desarrollo de la autonomía y de la independencia en el desarrollo de conocimiento del alumno, promoviéndose la autoevaluación permanente en interacción constante con el docente.

- Permite la construcción de espacios de reflexión y acción grupales, con la posibilidad de que se expresen los conocimientos, experiencias y opiniones de los diversos participantes, específicamente a través de los foros de discusión y los trabajos grupales.

- Facilita actualizar los contenidos didácticos y realizar un seguimiento del aprendizaje del alumno.

- Posibilita la interacción simultánea entre profesores y alumnos, formando foros de discusión y enseñanza con preguntas y respuestas vía online con sistemas auditivos y visuales (por ejemplo, el sistema Elluminate ${ }^{1}$.

\section{Problemas de la enseñanza virtual}

Sin embargo, la enseñanza virtual también posee una serie de dificultades, por lo que no se debe pensar que puede sustituir al resto de los modelos educativos en cualquier contexto. El modelo presencial clásico posee algunas características que la enseñanza virtual no puede suplir, como es el contacto personal y la instantaneidad de las respuestas a interrogantes. Entre los problemas de la enseñanza virtual se encuentran:

- La falta de contacto personal y la brecha digital provocada por los medios técnicos.

- La actitud de las personas que intervienen en el proceso. Los participantes pueden no estar acostumbrados a trabajar online. Se exige que el participante trabaje en forma independiente, se organice, se responsabilice y confíe en los beneficios pedagógicos de estas tecnologías para la educación.

- Actualmente no existen métodos confiables para determinar la identidad real de los usuarios de Internet, por lo que certificados de cursos online podrían obtenerse de manera fraudulenta.

- Podrían introducirse usuarios con intenciones maliciosas de interferencia.

- El proceso de aprendizaje centrado en el estudiante puede provocar desmotivación y abandono. Se torna necesaria la figura de un docente tutor o asesor que motive.

- Podría cometerse el error de aplicar simplemente

1 Ver sitio en Internet: http://www.elluminate.com/index.jsp 
procesos pedagógicos tradicionales, como "colgar" un documento informativo en Internet y llamar a esto "enseñanza virtual".

- Se necesita tanto la formación de docentes y alumnos en enseñanza virtual, como la presencia de personal técnico experto que asesore y ayude en Metodología Docente Virtual, Diseño Instructivo, Maquetación y Diseño Gráfico.

\section{Desafíos}

El conocimiento bioético comienza con el descubrimiento de que no existen certezas absolutas sino incertidumbres. Esto se logra mediante el cuestionamiento continuo, la crítica, la reflexión, el debate, el diálogo y la interacción con otros que permite encontrar otras formas de interpretación de la realidad. Por ello, aparte de adquirir conocimientos y habilidades específicas, es importante el perfeccionamiento de actitudes y la adquisición de un carácter ético.

A través de la enseñanza virtual se busca un aprendizaje experiencial, independiente e interactivo; una conversación didáctica guiada con materiales representativos del estado actual de la disciplina. Es necesario, entonces, proponer objetivos educacionales que contemplen las distintas competencias de los participantes y proporcionar estrategias de aprendizaje y recursos académicos que faciliten, motiven y estimulen al estudiante a autoaprender y a interactuar con la información, con los otros alumnos y con su docente.

Por lo tanto, en la enseñanza virtual se presentan los siguientes desafíos:

- Elaborar cursos online que faciliten la comunicación interpersonal y no sean simplemente un mero soporte de información.

- Que la enseńanza sea eminentemente práctica.

- Desarrollar actitudes y habilidades de comunicación.

- Lograr que el docente actúe como guía, asesor, tutor y evaluador del aprendizaje del alumno, haciendo uso de diferentes materiales didácticos y creando situaciones que favorezcan el aprendizaje.

- Formar comunidades de aprendizaje para posibilitar un aprendizaje interactivo entre el docente y alum- no y entre los alumnos, lo que podría denominarse "formación de aulas virtuales".

- Capacitar un nuevo tipo de docente con las funciones de asesor y tutor y la capacidad de trabajar de forma inter $y$ transdisciplinaria. Entre sus funciones principales se encontrarían: dirigir al alumno en la adquisición de conocimiento y favorecer su estudio independiente; orientar al alumno en torno a las actividades de aprendizaje; acompańarlo a lo largo de su trayectoria en el programa; realizar diagnósticos de sus necesidades, intereses y expectativas; promover en él estrategias de estudio y evaluar su progreso.

- Capacitar a los alumnos para adaptarse al modelo de autoaprendizaje, pues las nuevas generaciones conocen la tecnología y saben autoadministrar los contenidos que los profesores les entregan, no así las anteriores generaciones.

- Exigir de los participantes una constante reflexión, análisis, crítica y creatividad, que redunde en una formación de calidad.

\section{Conclusiones}

Si bien la modalidad de enseñanza virtual promete innovar fuertemente en el panorama del aprendizaje presente y futuro, cuenta en este momento con muy poco control de calidad, debido a su carácter transicional. Prácticamente, el valor de un programa de educación se deduce a priori por la calidad de la institución que lo promueve o por responder a las demandas y necesidades sociales. Existe poca supervisión en cuanto a los niveles de fiscalización, de control de asistencia, de tareas y foros. A medida que mejore la calidad de los programas, por la incorporación de diseños innovadores de aprendizaje, la modalidad de educación virtual podrá ser más efectiva en sus objetivos pedagógicos.

Debido a que la enseñanza virtual no puede suplir ciertos procesos que ocurren en la enseñanza tradicional presencial, se recomienda el uso de modelos de aprendizaje semipresenciales o de combinación mixta presencial y no presencial. De este modo, se puede mejorar el aprendizaje del alumno en distintas situaciones y favorecer la educación continua de personas que no tienen el tiempo para un aprendizaje tradicional. 


\section{Referencias}

1. Instituto Internacional para la Educación Superior en América Latina y el Caribe. La Educación Superior Virtual en América Latina y el Caribe. IESALC, 2004. (Sitio en Internet) Disponible en http://www.iesalc.unesco.org.ve

2. Ormrod JE. Educational Psychology: Developing Learners (Fourth Edition). Upper Saddle River, NJ: Prentice-Hall, Inc.; 2003: 227.

3. Lolas F. Bioética. Santiago de Chile: Editorial Universitaria; 1998: 33-42.

Recibido: 18 de enero de 2008

Aceptado: 8 de febrero de 2008 